\title{
INTEGRATING INJURY SCREENING WITH MEASUREMENT AND MONITORING: A CONCEPTUAL APPROACH USING A PATIENT Global Assessment of Body AND Limbs Scale
}

\begin{abstract}
Purpose: To develop a conceptual model for patients with musculoskeletal injuries that relates Injury Screening to Measurement and Monitoring (ISMAM). Screening scores would predict quantifiable outcomes on a proposed Global Assessment of Body And Limbs (GABAL) composite scale. The scale would define status as a percentage of pre-injury capacity using quantitative and qualitative self report outcome measures combined with work and life status data.
\end{abstract}

Background: Screening questionnaires use psychosocial yellow flags and activity limitation to identify potential chronic patients. Outcome measures provide clinical evidence by establishing patient status and assessing intervening change. Independently developed, definitive statistical links between these established concepts are yet to be determined.

Description: The ISMAM components are integrated using a graph of time versus score on the GABAL-scale with initial screening predicting recovery time to a designated pre-injury percentage level. Actual status would be assessed through initial then subsequent sequential measurements with GABAL-scale scores enabling trendline analysis to verify if the rate of actual recovery coincides with that predicted by screening.

Observations: Face and content validity are apparent because validated screening tools are available and the required components for the GABAL-scale would be existing validated outcome measures and quantifiable data.

Conclusions: This model should provide a practical method of integrating screening and global measurement that facilitates communication across agencies and professions. A clinical research trial to validate the ISMAM concept has been initiated.

KEY WORDS: EVIDENCE BASED PRACTICE, OUTCOME MEASURES, MUSCULOSKELETAL, ASSESSMENT. et al 2005; Stratford and Riddle 2005) by quantifying the patient's status and any change over time. A more recent complementary trend has seen the introduction of prospective screening using a biopsychosocial approach (Linton and Boersma 2003; WC-NSW 2006). This can determine the potential risk of chronicity and assist early identification of patients likely to have increased absenteeism and a delayed return to work; factors that can lead to increased total medical, rehabilitation centred focus (Sackett et al 1996). This concept, in particular, is emphasised in the management of compensable/insured musculoskeletal patients. Evidence is advocated through the use of a standardised systematic approach (APA 2003) which encourages the measurement and monitoring of patients by means of recognised Patient Report Outcomes (PROs). These reflect all three components of the EBP definition (Herbert

\section{CORRESPONDENCE TO: \\ Philip Gabel \\ University of the Sunshine Coast, Queensland, Australia PO Box 760 Coolum Qld 4573 \\ Tel: 61 (0) 75446 4444; \\ Fax: 61 (0) 754463344 \\ Email: cp.gabel@bigpond.com}


and workers compensation costs (Hurley et al 2001; Linton and Boersma 2003).

A logical progression is the integration of the screening and measurement concepts to relate 'Injury Screening' to 'Measurement And Monitoring' (ISMAM). Theoretically, a definitive relationship should exist between the two components whereby screening would provide a quantifiable prediction of the eventual outcome measurement. This could be a specific value, a percentage of pre-injury status or the time required to reach either of these levels. However, for this to occur the existing PRO scales of regional, quantitative and qualitative status alone would not suffice. A new PRO scale would be required that demonstrates a Global Assessment of Body And Limbs (GABAL) and integrates both existing qualitative and quantitative measures and specific levels of work or activities of daily living. To facilitate peer acceptance and ensure face and content validity, this GABAL-scale would need to be a composite of existing validated quantitative and qualitative PROs. Existing measures would need to be progressed, supplement and integrated into a single tool that would also include quantification of work and life attributes that are of critical importance to patients (Sackett et al 1996), professional groups (APA 2003) and insurers (WC-NSW 2006). The final validated GABAL-scale would be a PRO that exhibits simplicity for patient completion, therapist scoring and case manager interpretation.

This paper proposes five specific components as the preliminary basis of the GABAL-scale. Recommended individual variables are selected based on current models of Australian insurer and professional organisations accepted outcome criteria (APA 2003; WC-NSW 2006) and on key recommendations from reviews on the use of outcome measures and patient management (Ritchie 2001; Rossignol 2003; Stratford and Riddle 2005). The proposed five components are: 1) quantitative: self report status; 2) qualitative: self report status; 3 ) hours of work or daily routine; 4) salary, earnings or satisfaction recompense and 5) duties or activities performed. The three latter components would each be quantified as a proportional percentage of their preinjury level. Whether these five composite components truly reflect global status is currently unknown, however they would be clarified through further clinical research. This would provide justification for adding different components and retaining or removing those proposed. The specific ratio or weighting of the individual component scores to form the final composite total similarly will require validation through further research. The final components of such a composite GABAL-scale will reflect a means by which the injured individual's status would be defined in a global manner as a proportion of their pre-injury status. A global assessment measurement scale has the potential to meet the demands of government and corporate health services by documenting the outcome of clinical care (APA 2003). It provides external objective measures to substantiate clinical rationale (Ritchie 2001; Bardin 2003) and justify intervention.

\section{BACKGROUND}

The provision of EBP through the use of outcome measures as advocated by insurers, third party payers and professional organisations enables the clinician, case manager and potentially any external auditor to rapidly quantify and establish an individual patient's status. In this way the outcome of any intervention can be assessed with a view to justifying of its use and evaluating the costs involved (Ritchie 2001; Bardin 2003). It is accepted that PROs measure outcomes and the change in status that has occurred in the subsequent interval between measures. Such change is not the result of an isolated intervention, but is rather related to multiple factors that can include the conditions' natural course, placebo effect and other components (Herbert et al 2005). The effectiveness of interventions in an interim period can best be determined and justified by the use of PROs (Stratford and Riddle 2005; Campbell et al 2006). These tools enable the measurement of multi-factorial changes relevant to the chosen intervention and provide evidence and accountability for treatment and standards of care (Ritchie 2001; APA 2003; Bardin 2003). Outcome measures provide objective evidence that is clinically significant and patientcentred as well as being impartial, valid, reliable and responsive (Bardin 2003; Herbert et al 2005). Furthermore, such evidence is becoming an integral part of responsible clinical management and is advocated by professional groups, insurers, governmental bodies and third party payers.

By contrast, the use of screening questionnaires seeks to determine psychosocial yellow flag signs and, with the combined presence of physical function limitations, to identify the potential risk of chronicity (Linton and Boersma 2003). Their use has gained increased popularity as a means of early identification of patients likely to require longer recovery time, ongoing treatment and higher costs. At present the focus of screening tool development and validation has been in low back pain (LBP) populations with tools such as the Orebro Musculoskeleatal Pain Questionnaire (OMPQ) (Linton and Boersma 2003) being shown to have predictive capacity in both workers compensation and national health patient groups (Hurley et al 2001; Linton and Boersma 2003). However, only limited work has been performed to validate a modified OMPQ tool in populations that encompass all musculoskeletal injuries (Dunstan et al 2005).

The two concepts of screening and measurement have been developed independently and a definitive statistical link between them has yet to be established. This integration would be simplified if consistency was present in the format of existing PROs. Despite the advances within PRO research, most tools have been developed in relative isolation. This is demonstrated when existing functional assessment tools from different body regions are compared. Examples include spinal PROs such as the Oswestry, Roland Morris, Neck Disability Index or Functional Rater Index and extremity tools such as the Lower Extremity Functional Scale or the Upper Limb Functional Index and Disability Arm Shoulder and Hand. When compared, these tools show minimal consistency in item variables and limited continuity in tool format preventing integration into a single holistic patient-focused system.

An alternative to these quantitative tools with pre-selected item constructs are qualitative measures such as the Patient Specific Functional Scale (Stratford and Riddle 2005) and derivations of this concept like the Patient Specific Index (Gabel et al 2006). Tools with this validated concept use item 


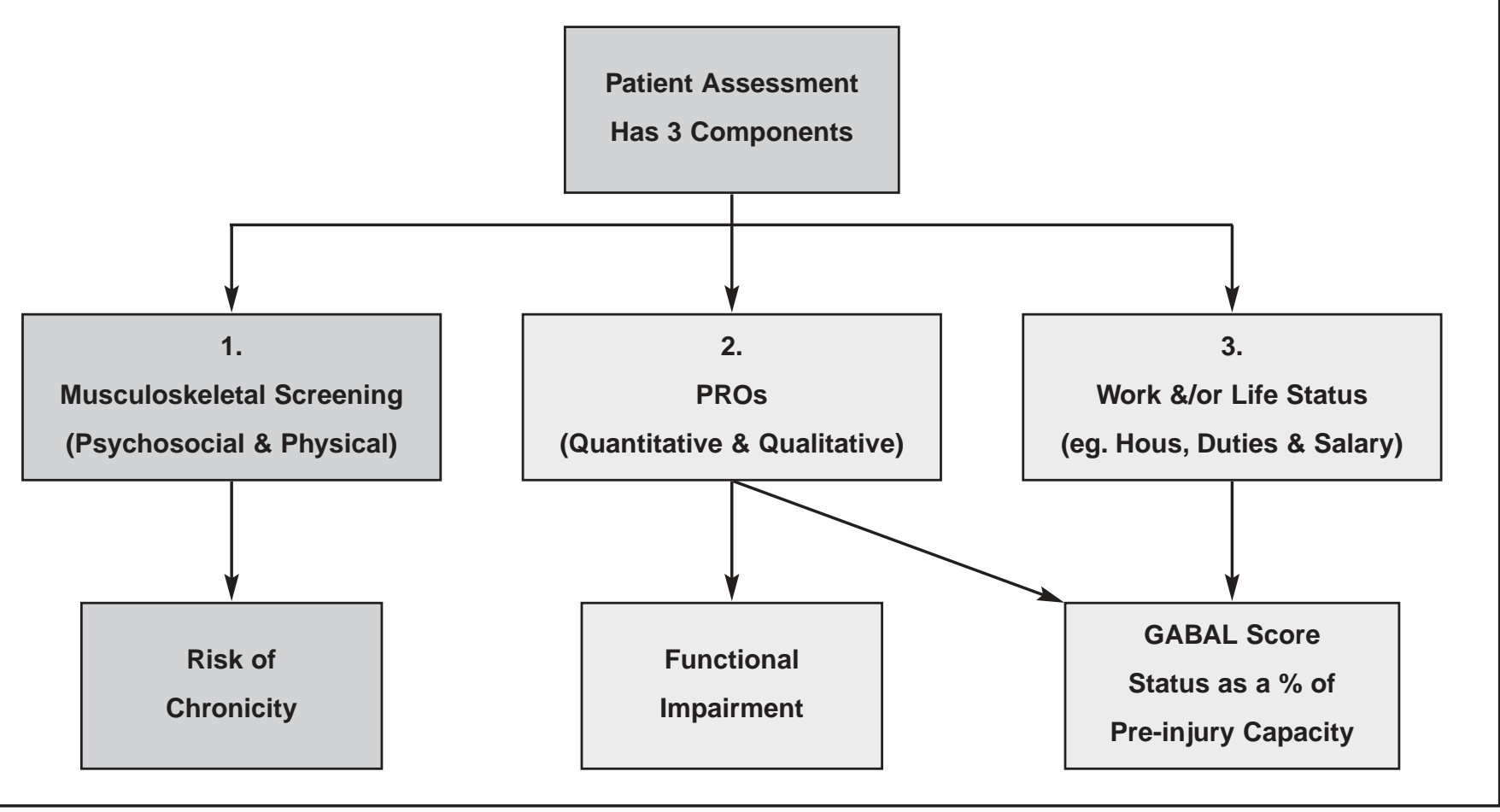

The patient at initial assessment completes 3 components:

1) Screening: a single use item assessing the risk of chronicity and predicting either impairment level or time to reach it.

2) PROs: quantitative and qualitative PROs providing functional scores which contribute to a composite GABAL-scale.

3) Work / Life status data: measures of current levels of performance of normal daily or work related activities, such as hours, duties or salary, measured as a percentage of pre-injury status and contribute to a composite GABAL-scale.

Measurement assessments would be repeated at 1, 2 or 4 weekly intervals for PRO and Work / Life data to provide their individual component scores and contribution to the composite GABAL-scale score.

variables determined by the patient to enable functional assessment in all regions. The concept is well received and endorsed by both professional, peer and governmental organisations such as Veterans Affairs agencies and Workers Compensation groups (APA 2003; WC-NSW 2006). Because these tools are 'patient specific', the selected item variables cannot be used for comparison between patients or across patient populations (Stratford and Riddle 2005). To overcome this difficulty, different research groups have pursued the development and validation of a series of quantitative tools with consistency of format and item variables across the three primary areas of the limbs and spine. These have included the work by Stratford and colleagues who developed the Upper and Lower Extremity and Back Pain Functional Scales (Stratford and Riddle 2005) and by Gabel and colleagues who developed the Upper and Lower Limb and Spinal Functional Index tools (Gabel et al 2006). Another alternative is Computer Adaptive Test (CAT) and Item Response Theory (IRT) that provides scaled quantitative outcome scores across each of the body regions. Such tools with item and format consistency enable direct comparison between patients and across different population groups.

The ISMAM model emphasises the need to demonstrate that beneficial outcomes are due to the therapeutic intervention itself or the natural progression of injury, not chance or other coincidental occurrences (Ritchie 2001; Stratford and Riddle 2005; Gabel et al 2006). It supports scientific evaluation through objective, patient-provided criteria (Sackett et al 1996; Herbert et al 2005). It is important however, that the patient be considered holistically, as injury affects both body and mind. Consequently, any tool for prediction or measurement of health status must consider essential domains that describe health and function within a multidisciplinary approach for individuals of all ages. It must be consistent with the World Health Organisation's (WHO) 'International Classification of Functioning' (ICF) whose domains include impairment, activity limitation, participation restriction, wellbeing and distress (WHO 2001). This is achieved through the selection of PROs that have demonstrated these criteria either through research findings or within the context of 'health related quality of life' during their initial development and validation process and still reflect these findings in their final scoring methodology (Stratford and Riddle 2005; Gabel et al 2006).

\section{DESCRIPTION}

The ISMAM concept ensures that initial screening is cross referenced with concurrent then subsequent outcome measurements. These measures indicate status on a common GABAL-scale expressed as a percentage measure of the patient's pre-injury capacity (Figure 1). It is a proactive individualised approach with initial one-off use of a standalone generic screening tool that would indicate the risk of chronicity using continuous data. Concurrently, additional information from repeated measures of qualitative and quantitative outcome tools would provide ordinal data which would then be combined with work and life status measures, such as hours, duties and salary. Together these variables would provide the score on the composite GABAL-scale. Component variables would be represented in percentage values of the individual 
patient's normal pre-injury capacity with their ratios being determined by research and statistical analysis. This would provide a single generic value on the GABAL-scale that reflects global status as a percentage of pre-injury capacity. This score would be measured every one to two weeks in the initial acute stage as change in this period due to treatment and natural healing is more rapid. Once the sub-acute to chronic stage is reached, where change is anticipated to be slower, then repeated GABAL-scale measures would be every two to four weeks until discharge.

The flow of the ISMAM concept and its sequencing is shown in the algorithm in Figure 2. The screening score predicts future recovery time to a designated preinjury status level whilst the GABALscale score quantifies measurement of the existing status. With repeated outcome measures a graphical sequential representation of this status can be produced with the horizontal axis representing time and the vertical axis the 'GABAL-scale' which indicates the global preinjury capacity. A hypothetical example using monthly measures and both linear and logarithmic trendlines to forecast potential progress is shown in Figure 3. A trendline can be established by the third measurement that will predict an individual clinical pathway and anticipated progress. It would also propose an expected point of recovery to a designated pre-injury capacity.

It is hypothesised that a link will be found between the scores on a suitable screening tool and the time taken to achieve the level indicated. The trendline extrapolation from the actual measures would indicate if actual recovery time and status would coincide with the specified level initially predicted by the screening tool. Using logarithmic trend extrapolation in Figure 3, the anticipated $95 \%$ level of pre-injury capacity is 7.5 months - an estimate reinforced by the fourth and fifth measure. Other predesignated levels, for example $90 \%$, could be similarly used in which case the prediction time of 6.75 months would be found. By contrast, if a linear trendline were used then the $95 \%$ and $90 \%$ recovery levels would be 5.75 and 5.48 months respectively. It is anticipated that future research will determine the critical GABAL-scale score that shows a statistical link with the initial screening score and whether linear, logarithmic or some other calculated trendline provides the best predictive model. Once that link is determined then it will enable a target prediction recovery level to be placed on the graph as a reference and comparative point for the trendline extrapolation. This process enables the recovery time to a designated level to be estimated and the progress pathway to be continuously monitored. Subsequently, the estimated costs of the recovery (based on the known weekly salary and medical expenditure) can be anticipated and determined from the graphical extrapolation and compared to that predicted from screening.

Figure 2: Detailed Algorithm for the process of Injury Screening Measurement and Monitoring (ISMAM).

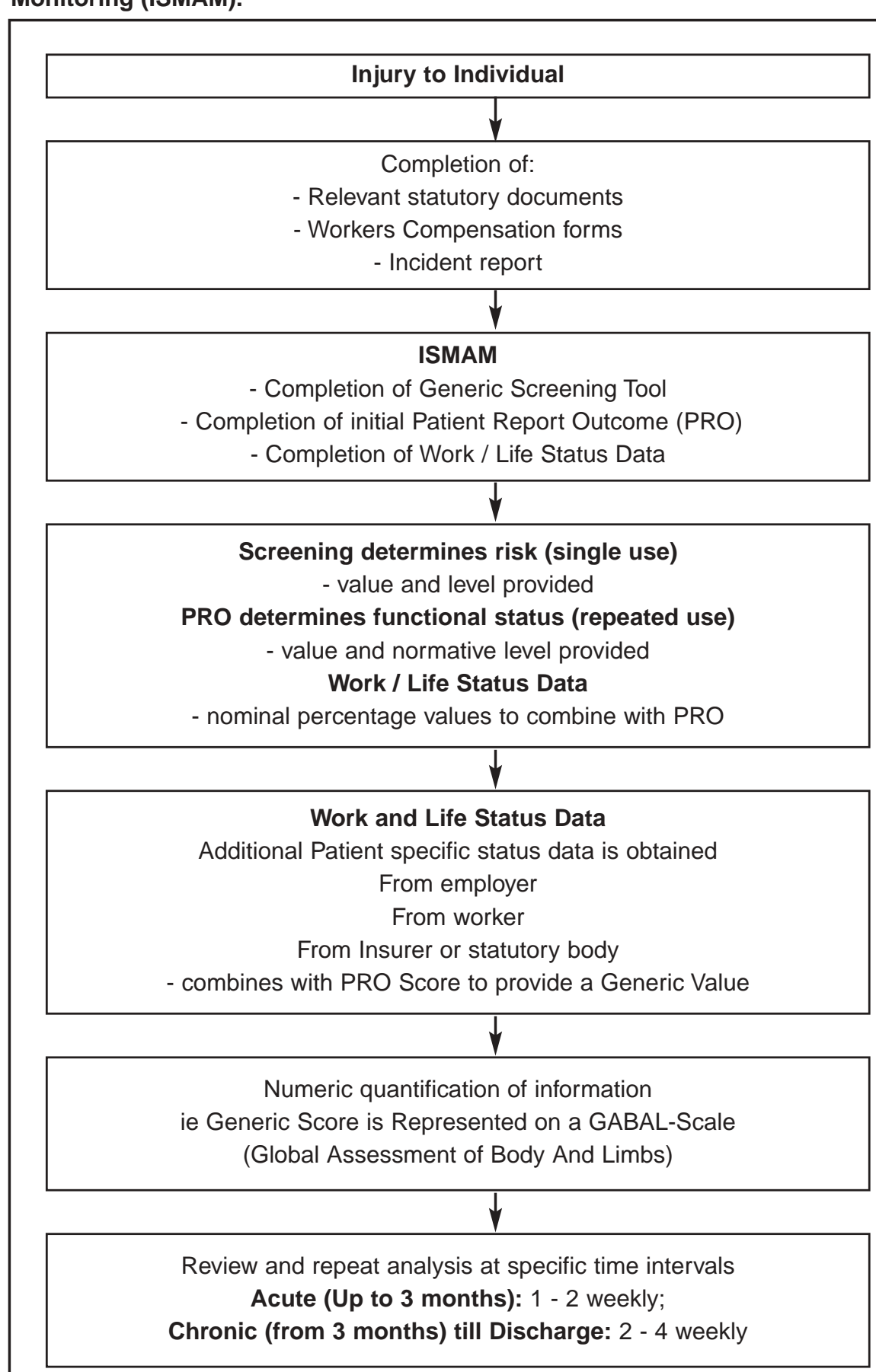

A case study that illustrates the ISMAM concept, using a modifiedOMPQ and the GABAL-scale for a distal radius fracture, is provided (Figure 4). This patient was involved in a motor accident and initially placed in an Intensive Care Unit (ICU). Measurements were made at weekly to fortnightly intervals over the initial two months and then reduced to monthly until discharge. Screening with the modified-OMPQ tool (Dunstan et al 2005) provided a score of 128 points which predicted more than 28 days off work (Linton and Boersma 2003; Dunstan et al 2005). The GABAL-scale 
was constructed using the Upper Limb Functional Index and Patient Specific Index (Gabel et al 2006) and patient reported percentage values for work hours, salary and duties compared to pre-injury levels. These were combined in at an arbitrary ratio of 40:15:15:15:15 respectively for the five values to provide the composite score. A consistent improvement over the first four weeks is shown with a rapid improvement on return to work at five weeks, then a gradual palteauing over the subsequent eight weeks. Intuitively this visualised progress illustrates the anticipated clinical recovery from such an injury (Stratford and Riddle 2005; Gabel et al 2006). As a case example the concept is demonstrated. It is anticipated that future research will determine the relationship between screening scores, such as 128 in this example, and if it will equate to a specified score range on the GABAL-scale, such as 90 or $95 \%$. Alternatively it may predict the time required to reach these levels - such as 13 weeks to the $90 \%$ level.

The ISMAM model may offer an objective standard utilising validated assessment tools which is in contrast to most medical models currently used in the various compensable systems (Ritchie 2001; Bardin 2003; Linton and Boersma 2003; Rossignol 2003). Furthermore, the ISMAM model may also provide a prospective predictive assessment of risk and recovery that is continuously and individually quantified and adjusted throughout the recovery period from initial presentation through to final discharge. The use of screening and measurement PROs provides a two-stage process with separate values for musculoskeletal injury prediction and assessment. The former will alert those involved in the patient's management to the potential risk of chronicity whilst the latter will provide a consistent global and regional picture of the injured individual's qualitative and quantitative status when compared to their pre-injury level.

\section{OBSERVATIONS}

Critical to any new concept and scale is its validity. In the development and theoretical stage it is important that face and content validity be apparent whilst construct and criterion validity, along with the practical characteristics - such as completion and scoring time and psycho- metric properties - such as reliability, error range and responsiveness, are established through clinical research. With the ISMAM concept, face and content validity appear evident as all existing PRO tools required would be validated measures and additional data relating

to work or daily duties is rapidly quantifiable from the patient (Dawson et al 2002). In this way the GABAL-scale would similarly exhibit face validity as it would be a measure of global status (Rossignol 2003; Gabel et al 2006). Its content, such as the five proposed

Figure 3: Sample ISMAM chart illustrating format and prediction through the use of trendlines.

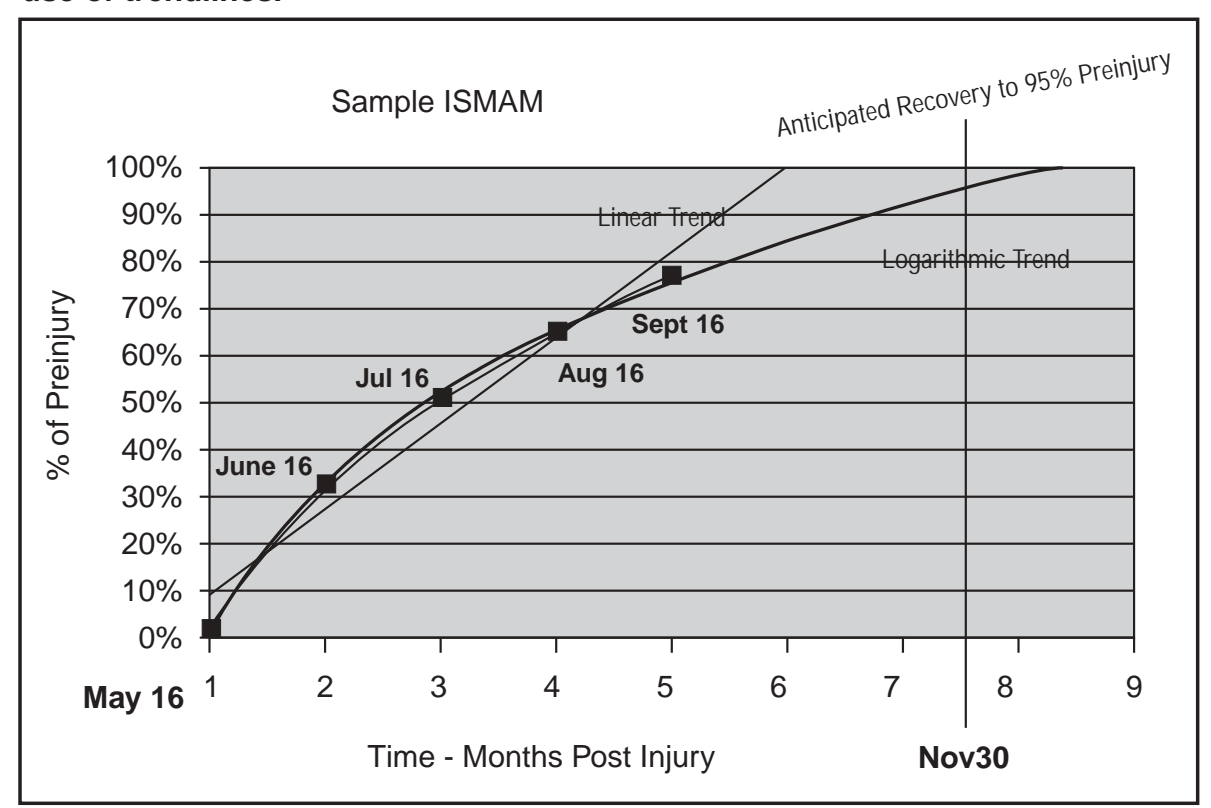

$\mathrm{X}$ Axis = Time in months with repeated measures, initially at baseline, then monthly; $\mathrm{Y}$ Axis = A composite GABAL-scale representing the patient as a percentage of their pre-injury capacity.

Composite GABAL components are assumed as being $100 \%$ values at their pre-injury level.

Figure 4: Case Example using an ISMAM Chart for the upper limb of a motor accident patient initially in ICU.

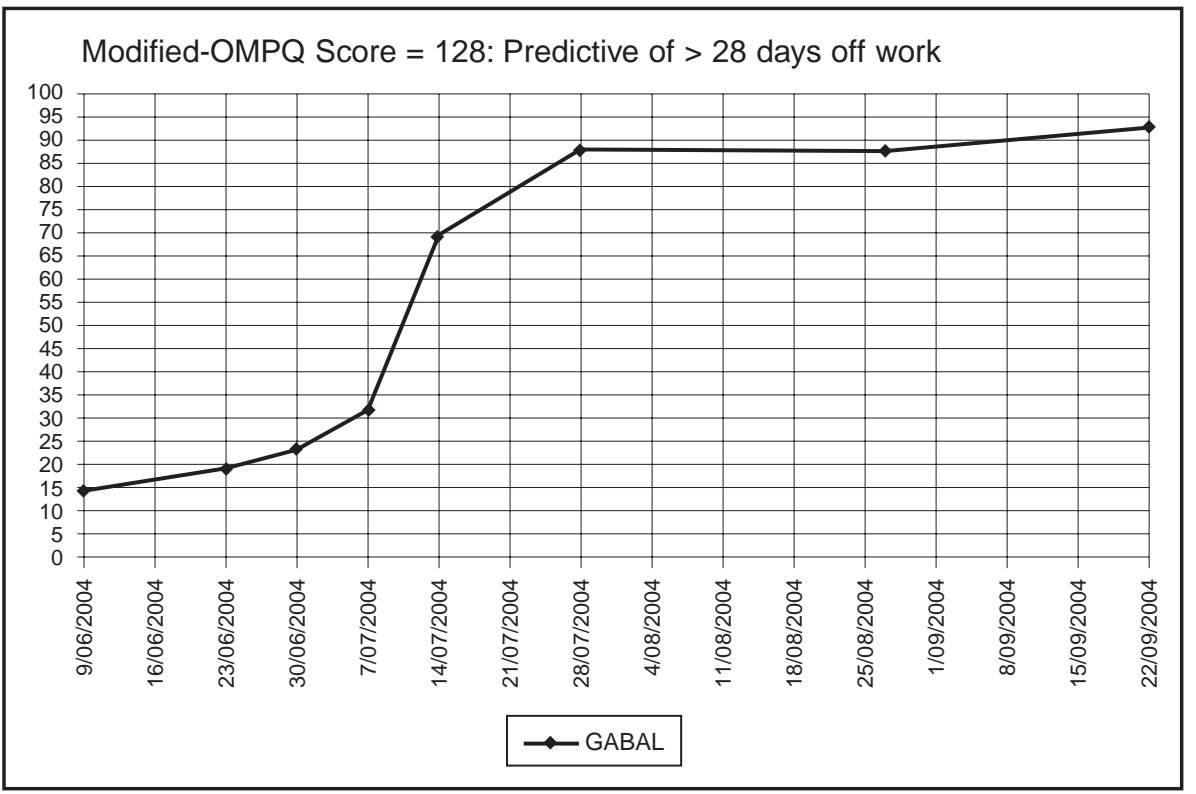

$\mathrm{X}$ Axis $=$ Time in weeks with repeated measures at baseline, then week 2, 3, 4, 5, 7, 11 and 13;

$\mathrm{Y}$ Axis = GABAL-Scale: a representation of the patient as a percentage of pre-injury capacity.

Pre-injury capacity is assumed as $100 \%$ or with only maximum values in each of the 5 contributing components.

Patient returned to work on 14.7.04. 
components, would demonstrate content validity as they each portray the constructs of interest and have been independently validated through existing research (Stratford and Riddle 2005).

The proposed individual components that form this new scale may be contentious. Included variables and their specific weighting ratio to each other will require validation through clinical research. It may be argued that the proposed five selected composite variables are not representative of a global score as not all aspects of recognised general health scales, such as the SF-36 or the subcomponents of the WHO ICF (WHO 2001) are included. Such criticism might be valid, however should not detract from the merits of the concept. There is currently significant evidence and literature support for the view that quantitative regional PROs are the most specific and relevant measurement for determining regional impairment status and that they reflect HRQOL and holistic functional activity (Rossignol 2003; Stratford and Riddle 2005; Campbell et al 2006; Gabel et al 2006). In addition there is ample scope for specific individual expression within the proposed GABAL-scale through the use of the qualitative tool aspects. The data of hours, duties and recompense are considered three essential criteria for return to work capacity by the insurance industry (WC-NSW 2006). This view is supported by occupational assessment standards (Dawson et al 2002) and review publications (Rossignol 2003).

Consideration of the algorithm (Figure 2) and components of the ISMAM concept (Figure 1) enable the potential demonstration of several outcomes and forms of external evidence. These include: the stand alone scores from the screening tool and both the qualitative and quantitative outcome tools; their composite GABAL-scale score; the measured values and the extrapolation trend-graphing for clinical pathways. This extrapolation would enable costing to be estimated by using known weekly expenses (including employer, medical and rehabilitation costs) multiplied by the predicted number of weeks to the designated recovery level. The predictive capacity of screening tools has been demonstrated in LBP populations by several authors over recent years with an integrated consistency between screening and various outcomes including absenteeism (Linton and Boersma 2003) and failure to return to work at 6 months post injury (Hurley et al 2001). Further research has developed this through the investigation of any musculoskeletal injury population (Dunstan et al 2005). This predictive capacity of screening will have significant implications for the management of compensable patients. It will also become a critical area in future investigative research for strategies in compensable injury claims management and early intervention.

To validate a final scale any argument for inclusion, exclusion or the weighting ratio of composite variables will require support from investigative patientspecific research trials. This paper and the case examples presented (Figures 3 and 4) propose and illustrate the concept of the model, the specific content will evolve with further research. It is anticipated that the ISMAM concept, using a composite GABAL-scale, will possess the essential psychometric properties required for demonstration of a robust and valid model.

\section{CONCLUSIONS}

This model provides a simple, clinicianfriendly method of integrating screening with global status measurement. It has the potential to facilitate communication between agencies and health professions. Demands for evidence from insurer, government agency, professional and patient groups continue to increase. These demands can best be met with a comprehensive integrated approach that provides prediction, outcome measurement, costing and accountability. The conceptual ISMAM model would satisfy these requirements as it is a proactive approach with patient focus and fulfilment of EBP requirements. It demonstrates risk and the generalised overall status of the injured individual by summarising current functional status, related capacity and predicting recovery time and subsequent costs. The model can be further developed and researched to provide a means of patient assessment and monitoring that is simple, effective and acceptable to all stakeholders. A clinical research trial to determine the model's viability has been initiated by the Centre for Healthy Activity Sport and Exercise at the University of the Sunshine Coast in Australia.

\section{REFERENCES}

APA: Australian Physiotherapy Association 2003: Policy on outcome measures and treatment justification. Melbourne:. http://apa.advsol.com.au/ members/documents.

Bardin LD 2003. "Physiotherapy and low back pain - Part III: Outcomes research utilising the biopsychosocial model: psychosocial outcomes." South African Journal of Physiotherapy 59(2): 16-24.

Campbell H, Rivero-Arias O, Johnston K, Gray A, Fairbank J, Frost H and UK MRC SST 2006. "Responsiveness of objective, disease-specific, and generic outcome measures in patients with chronic low back pain: an assessment for improving, stable, and deteriorating patients." Spine 31(7): 815-22.

Cook K, O’Malley KJ, Roddey TS. Dynamic assessment of health outcomes: time to let the CAT out of the bag? Health Serv Res 2005;40(5-Pt 2):1694-711.

Dawson EG, Kanim LE, Sra P, Dorey FJ, Goldstein TB, Delamarter RB and Sandhu HS 2002. "Low back pain recollection versus concurrent accounts: outcomes analysis." Spine 27(9): 984-93.

Dunstan D, Covic T, Tyson GA and Lennie IG 2005 "Does the Orebro Musculoskeletal Pain Questionnaire predict outcomes following a work-related compensable injury?" International Journal of Rehabilitation Research 28(4): 369-70.

Gabel CP, Michener L, Burkett B and Neller A 2006. "The Upper Limb Functional Index (ULFI): Development and Determination of Reliability, Validity and Responsiveness." Journal of Hand Therapy 19(3): In Press.

Herbert RD, Jamtvedt G, Mead J and Hagen KB 2005. "Outcome measures measure outcomes, not effects of intervention." Australian Journal of Physiotherapy 51(1): 3-4.

Hurley DA, Dusoir TE, M. MS, Moore AP and Baxter GD 2001. "How effective is the acute low back pain screening questionnaire for predicting 1-year followup in patients with low back pain?" Clinical Journal of Pain 17(3): 256-263.

Linton SJ and Boersma K 2003. "Early identification of patients at risk of developing a persistent back problem: the predictive validity of the Orebro Musculoskeletal Pain Questionnaire.” Clinical Journal of Pain 19(2): 80-86.

Ritchie JE 2001. "Case series research: a case for qualitative method in assembling evidence." Physiotherapy Theory and Practice 17(3): 127-135.

Rossignol M 2003. "The management of low back pain." Occupational Environmental Medicine 60(9): 617 .

Sackett DL, Rosenberg W, Muir Gray JA, Haynes RB and Richardson WS 1996. "Evidence based medicine: what it is and what it isn't." British Medical Journal 312: 71-72.

Stratford PW and Riddle DL 2005. "Assessing sensitivity to change: choosing the appropriate change coefficient." Health and Quality of Life Outcomes 3(23): 23.

WC-NSW: Workers Compensation Authority of New South Wales - Australia 2006. Management of Soft Tissue Injuries: Clinical Guideline - Electronic Website Report. Sydney: Motor Accident Authority; 1.3.06.http://www.workcover.nsw.gov.au/MediaResources/MediaReleases/2006/270206.htmNew.

WHO: World Health Organisation: 2001. "ICF International Classification of Functioning, Disability and Health." www.who.int/classifications/en/ WHOFICFamily.pdf 
